\title{
Amathonte : les abords Sud-Ouest de l'agora - 2017
}

Ludovic Thély, Julien Adam, Marie-Hélène Barrière, Camille Castres, François-Dominique Deltenre, Antigone Marangou, Rachel Nouet et Cécile Rocheron

\section{OpenEdition \\ Journals}

Édition électronique

URL : http://journals.openedition.org/baefe/1257

DOI : $10.4000 /$ baefe. 1257

ISSN : $2732-687 \mathrm{X}$

Éditeur

ResEFE

\section{Référence électronique}

Ludovic Thély, Julien Adam, Marie-Hélène Barrière, Camille Castres, François-Dominique Deltenre,

Antigone Marangou, Rachel Nouet et Cécile Rocheron, « Amathonte : les abords Sud-Ouest de l'agora 2017 » [notice archéologique], Bulletin archéologique des Écoles françaises à l'étranger [En ligne], Chypre, mis en ligne le 26 novembre 2020, consulté le 15 décembre 2020. URL : http:// journals.openedition.org/baefe/1257 ; DOI : https://doi.org/10.4000/baefe.1257

Ce document a été généré automatiquement le 15 décembre 2020.

\section{(c) $(1) \odot$}

Le Bulletin archéologique des Écoles françaises à l'étranger est mise à disposition selon les termes de la Licence Creative Commons Attribution - Pas d'Utilisation Commerciale - Pas de Modification 4.0 International. 


\title{
Amathonte : les abords Sud-Ouest de l'agora - 2017
}

\author{
Ludovic Thély, Julien Adam, Marie-Hélène Barrière, Camille Castres, \\ François-Dominique Deltenre, Antigone Marangou, Rachel Nouet et Cécile \\ Rocheron
}

\section{NOTE DE L'AUTEUR}

Autorité nationale présente : Yiannis Violaris (Département des Antiquités de Chypre, District de Limassol)

Composition de l'équipe de terrain : Ludovic Thély, responsable d'opération ; Julien Adam et François-Dominique Deltenre, chefs de secteur ; Camille Castres, architecte ; Antigone Marangou et Cécile Rocheron, céramologues.

Partenariats institutionnels : École française d'Athènes, Ministère de l'Europe et des Affaires étrangères.

Établissements porteurs de l'opération : École française d'Athènes, Ministère de l'Europe et des Affaires étrangères.

Remerciements : La mission exprime sa reconnaissance à Marina Solomidou-Ieronymidou, directrice du Département des Antiquités de Chypre, et à Yiannis Violaris, archéologue responsable du district de Limassol, pour leur collaboration et confiance. Toute sa gratitude va à l'Académie des Inscriptions et Belles-Lettres, qui a honoré L. Thély du prix de la Fondation Paule Dumesnil pour 2017.

Données scientifiques produites:

Mission française d'Amathonte par l'EFA

Mission française d'Amathonte par l'Ambassade de France à Chypre 


\section{Introduction}

1 La fouille des abords sud-ouest de l'agora d'Amathonte a débuté en 2014, d'abord par une étude géomorphologique (sous la responsabilité d'Antoine Chabrol) ${ }^{1}$, puis par une série de fouilles archéologiques (2014-2017) : fig. $1^{2}$.

Deux résultats principaux peuvent être soulignés en préambule :

1. La zone du prétendu bassin intérieur, située entre l'agora et le port, constitue un espace de très forte densité urbaine, de l'époque hellénistique jusqu'à son comblement progressif et tardif. Des traces d'activités artisanales ont été découvertes, ainsi que des structures massives dont celles situées au Nord, qui donnent à penser qu'il pourrait s'agir d'entrepôts ou de boutiques ouvertes sur la rue principale Est-Ouest.

2. Le port intérieur, selon l'hypothèse présentée communément, ne se trouve pas dans cette zone (ce qui ne signifie pas qu'il n'a jamais existé), mais les relations avec la mer ont certainement intéressé le secteur: remontée de la nappe phréatique qui a contraint à un rehaussement progressif des structures au Sud; présence d'un niveau sableux pauvre en matériel et bordé d'une structure à l'Est.

Les travaux réalisés en juin 2017 ont porté essentiellement sur la fouille d'un nouveau sondage à l'ouest du secteur 02. Le secteur 01 a fait l'objet d'un nettoyage intensif et d'une poursuite de la fouille dans les sondages déjà ouverts en 2014-2016.

Fig. 1 : Vue aérienne de la fouille à la fin de la campagne 2017.

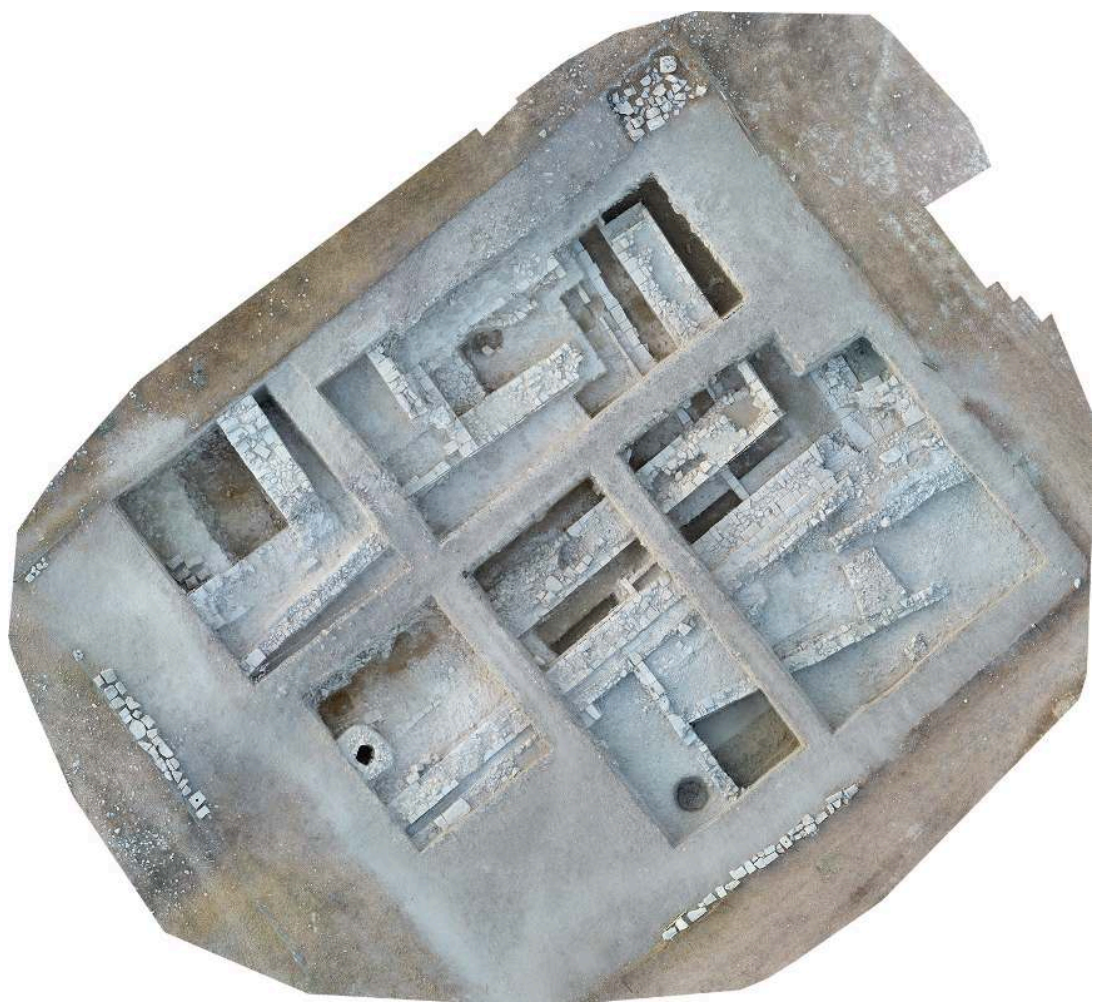

L. Thély / EFA. 


\section{Secteur 02}

4 La première journée a été consacrée au nettoyage de début de campagne des deux secteurs. L'état de conservation des structures demeure bon, les couches exposées ont parfois souffert par endroit, soit en raison de l'exposition permanente au soleil (couches très sèches et dures) ou au contraire de l'exposition aux intempéries (certaines bermes se sont délitées et ont partiellement contaminé certaines couches). En revanche, dans le secteur 02, un autre phénomène a été observé: à l'ouest d'UC 1001, le nettoyage, interrompu pour des raisons pratiques, a révélé la présence d'une couche argileuse extrêmement humide qui se trouvait elle-même sur une couche de sable et de petits coquillages. Nous avons formulé l'hypothèse d'une adaptation des structures au problème de la remontée de la nappe phréatique. Le niveau du terrain a peut-être été élevé progressivement et peut-être que certaines structures ont été abandonnées en raison de ce phénomène. Cela demeure néanmoins une hypothèse. La présence d'un fil différent sous les structures, y compris à certains endroits du Sect. 01, pourrait constituer un indice de ce phénomène. Une autre observation à signaler est la différence de niveau entre les assises d'UC 1001 entre les secteurs 01 et 02 . Il conviendrait d'analyser si cette différence est liée à la morphologie du terrain ou à une problématique particulière. En outre, un lien existe peut-être avec la réparation (reconstruction?) de l'angle d'UC 1001 (qui pourrait être l'indice d'une chronologie/ phase USM différente - l'inscription découverte en $2015^{3}$ n'est peut-être que le terminus post-quem de cette réparation/reconstruction).

5 Une monnaie a été retrouvée lors du nettoyage de surface. En outre, le délitement de la berme Ouest du Sect. 02 a révélé la présence d'un petit fragment architectural (moulure) et de la moitié d'un élément en pierre formant un demi-cercle, qui est demeuré dans la berme.

6 La fouille a débuté par l'implantation du sondage qui a été défini sous la forme d'un rectangle de $7 \times 8 \mathrm{~m}$, sans berme entre les sondages 2016 et 2017. L'extension du sondage est liée à la découverte d'un bloc apparemment en place au Nord de la zone lors du nettoyage général. Une première US a été identifiée et relevée : US 2201. Elle couvrait l'ensemble du sondage, était de couleur grise et très friable et hétérogène (même si elle peut présenter des différences de compacité par endroits). Elle contenait une quantité moyenne de matériel céramique très mélangé et généralement très fragmentaire. On a également découvert quelques fragments de verre et quelques clous. Cette couche s'étendait sur une profondeur variable selon les endroits. Au Nord, environ au centre du sondage, en bordure de sa limite, elle recouvrait plusieurs pierres taillées et alignées dont une était déjà visible lors du nettoyage. Les situations sousjacentes étaient assez semblables à ce qui avait été observé l'année précédente. En particulier, on a noté la présence (à Sud-Ouest) des traces de la fosse de 2016 (US 2104 coupe US 2105), qui contenait des coquilles d'escargots, des blocs de grande dimension et une terre brune friable avec beaucoup de déchets organiques décomposés. On a procédé à un nettoyage général pour identifier les différentes US sous-jacentes. La structure déjà apparente ne s'étendait pas davantage au Sud mais se poursuit au Nord, dans la berme. Elle est parfaitement parallèle au segment Nord-Sud d'UC 2005.

7 La fouille d'US 2201 a révélé une situation complexe, qui résulte très probablement d'une opération moderne de rejet (fig. 2). Nous avons identifié quatre US différentes : US 2202, compacte et brun clair, sur les deux tiers du sondage à partir du Nord; 
US 2203, une concentration de pierres de moyenne dimension au Sud-Ouest (il s'agit probablement d'une fosse) ; US 2205, avec des pierres de moyenne dimension, une terre noire très friable et des coquilles d'escargot ; US 2204, qui s'étend au Sud, de couleur brune, plus compacte et composée de petites pierres. US 2203 coupait US 2202 et US 2204

Fig. 2 : Situation des US 2202-2205.

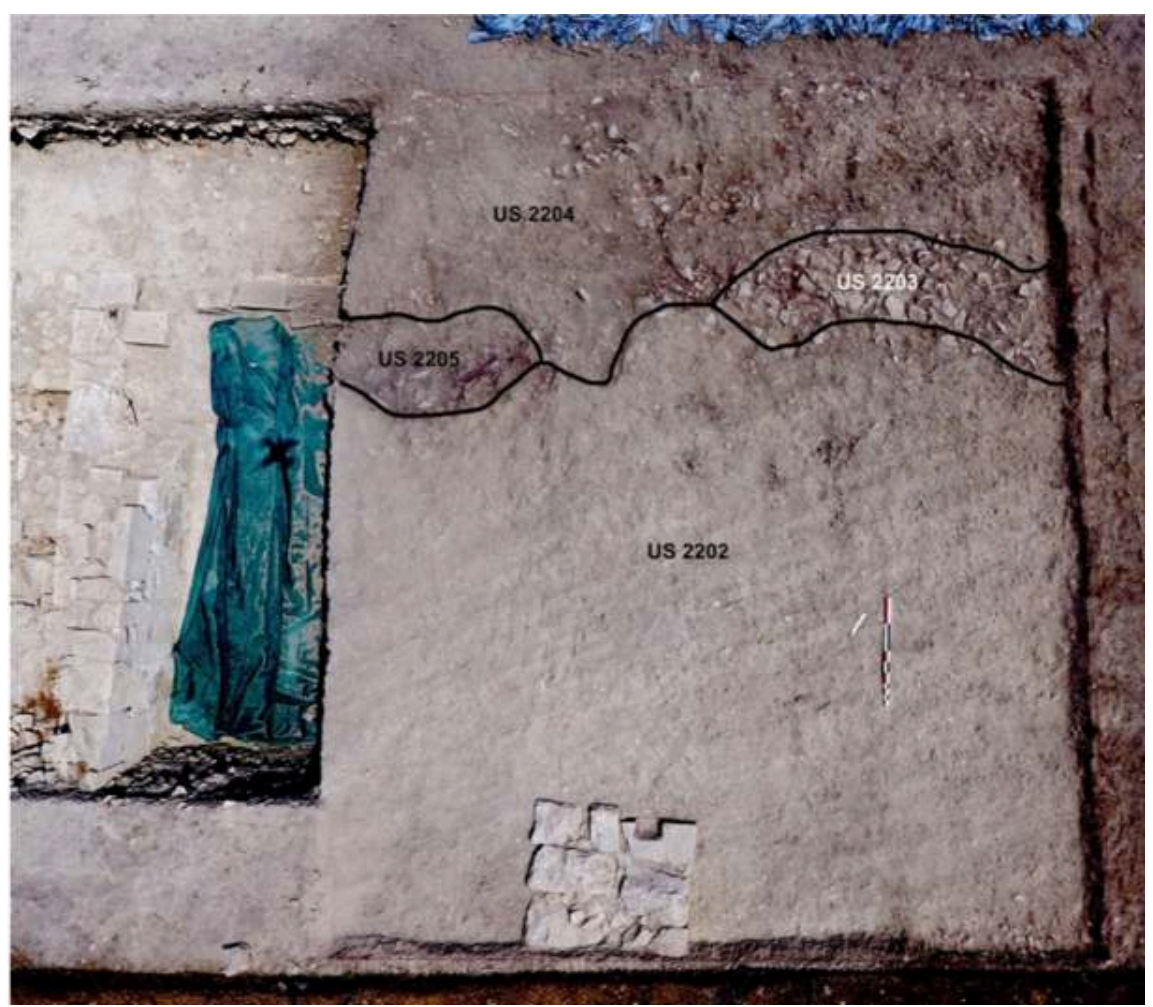

L. Thély / EFA.

8 Une clarification de la situation stratigraphique a alors été nécessaire. En réalité, la fosse US 2203 s'est révélée constituer une unique longue tranchée Ouest-Est. Le remplissage était assez incohérent: il était composé essentiellement de pierres de grandes et moyennes dimensions, d'une terre marron foncé-noir très friable et de coquilles d'escargots, concentrées en deux points (à l'Est et à l'Ouest du sondage). La forme de la tranchée laissait supposer une opération effectuée par la benne d'une pelleteuse. Plusieurs fragments architecturaux ont été découverts. La couche présentait également des fragments de mortier. La fosse coupait les US 2202 et 2207, ainsi que d'autres US sous-jacentes.

9 Après la fin du nettoyage et de la documentation de la coupe de fosse US 2204, on a procédé à la fouille de l'US 2202, qui était la première taillée par la fosse. Cette US s'étendait sur environ les deux tiers de la superficie Nord du sondage. Elle était de couleur gris-brun, de consistance compacte et comprenait quelques pierres de petites dimensions. Elle contenait une quantité notable de matériel céramique et amphorique. On y a également retrouvé des fragments de lampe (dont la moitié de l'une d'entre elles), un fragment de statuette en céramique, des os, deux ou trois fragments de verre et un clou en fer. Les limites sud de l'US étaient perturbées par les limites elles-mêmes peu claires de la fosse US 2203. On a constaté que le nouveau mur UC 2007 se 
poursuivait au Sud sous US 2202, en suivant une ligne Nord-Sud. La partie Sud était construite avec des blocs plus petits et plus irréguliers que la moitié Nord. Le mur présente en outre des traces de mortier sur son parement ouest, ainsi qu'entre les blocs de la seconde assise. La partie du mur conservée s'interrompt plus ou moins à la même distance au Sud que UC 2005. L'ensemble du sondage et de la butte semble donc avoir été perturbé par l'action dont résulte la fosse US 2203.

On a ensuite clarifié les rapports stratigraphiques autour de la coupe de la fosse (US 2204). Deux US jaunes sablonneuses (US 2205 et 2206) recouvraient une US brune plus compacte composée de pierres de petites dimensions (US 2207), respectivement à l'Est et à l'Ouest d'UC 2007 (fig. 3). En-dessous de l'US 2205 est apparue une US grise argileuse et très compacte, qui se superposait à l'US 2207 (situation visible dans la coupe de la fosse à l'Ouest du sondage). La position stratigraphique des US 2205 et 2206 était très claire : elles sont postérieures à toutes les autres US. On a donc procédé à la fouille de ces deux couches. Celles-ci ont se sont révélées abondantes en matériel (surtout céramique et amphorique), posséder de nombreuses inclusions de mortier, de fragments de pigments et de petits charbons (peu nombreux). Un objet métallique de forme allongée (AM 3922) a été découvert dans l'US 2205. Il est percé de trous. L'identification de cet artefact n'est, pour l'heure, pas encore claire. Les deux US, bien que présentant des similitudes, recouvraient des situations différentes. L'espace présent entre UC 2007 et la berme Est rendait cependant la compréhension de l'ensemble assez difficile.

Fig. 3 : Situation des US 2205-2207.

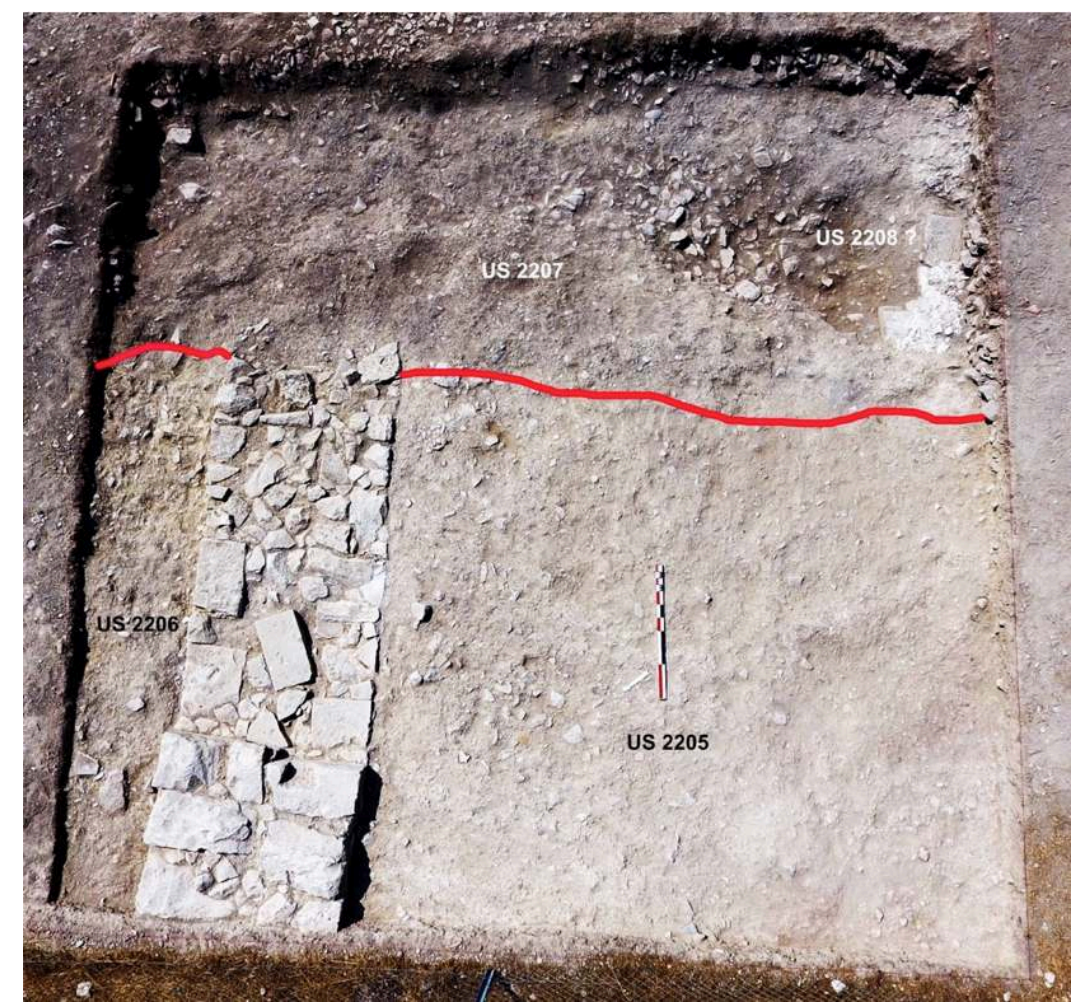

L. Thély / EFA.

L'observation de la situation dans la coupe de la fosse a également permis de découvrir une assise d'un autre mur, parallèle à UC 2007, constituant un espace (clos ?) dont la 
superficie est égale à celle de l'espace entre UC 2005 et 2007. Hors sondage, à l'Ouest, une pierre taillée affleurant nous a permis de supposer un plan hypothétique, qu'on propose en fig. 4. En jaune dans ce plan, la section Est-Ouest d'UC 2002, découvert lors des campagnes précédentes, doit être mise en relation avec les nouveaux murs mis en lumière en 2017. En bleu, la présence d'une structure est plus hypothétique en raison des limites du sondage de 2016.

\section{Fig. 4 : Proposition de reconstitution.}

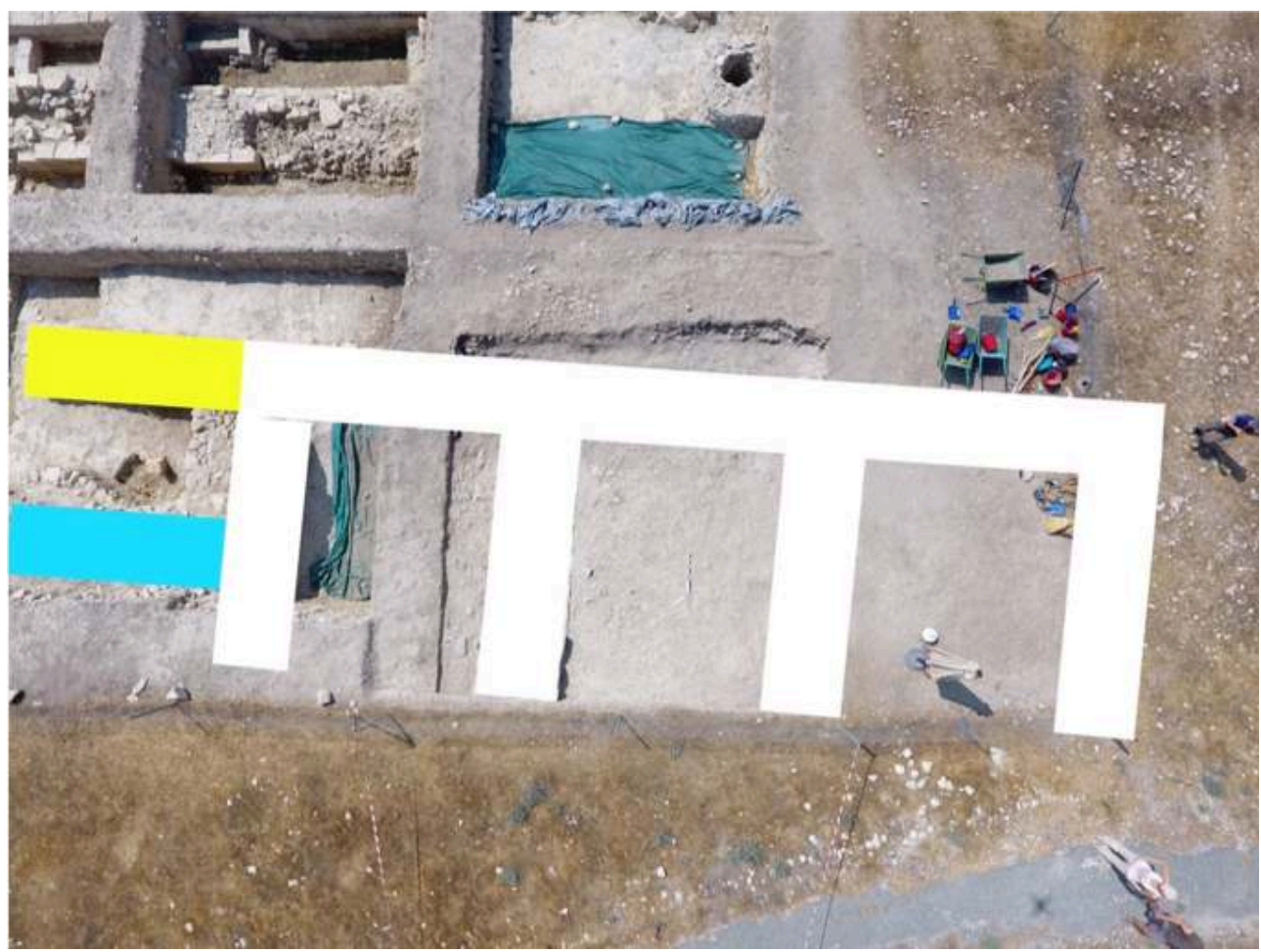

L. Thély / EFA.

La fouille d'US 2206 s'est révélée compliquée du fait du peu d'espace disponible entre le mur et la berme. La couche sous-jacente révélée par US 2206 était une couche argileuse gris clair, au pendage assez irrégulier. Elle se composait de nombreuses petites pierres et de fragments de mortier. Environ à 1,5 $\mathrm{m}$ de la berme Nord, un bloc taillé était posé à plat: il s'agissait probablement d'un élément du mur qui s'en était détaché et était ensuite tombé. La fouille de l'US 2205 a été également difficile du fait de son hétérogénéité. Elle a néanmoins révélé la présence d'au moins deux couches distinctes. L'une, argileuse et plastique, de couleur grise, et l'autre, jaune et plastique. Le nettoyage du parement Est d'UC 2007 a montré que son dernier tiers visible était composé de petites pierres irrégulières, grossièrement taillées, et correspondait à une ligne de coupe dans le mur qui était peut-être la trace d'une restauration ou de la fermeture d'un passage entre deux espaces. On a interprété ces différentes couches comme un remblai moderne.

13 En procédant à la fin de la fouille d'US 2205, la couche gris clair composée de petites inclusions de mortier et de fragments de charbon a été mise en lumière sous l'ensemble de la surface de l'US 2205, avec de nombreuses différences d'altitude. L'US 2205 recouvrait également au Nord une couche brune relativement compacte, granuleuse, 
composée de petites pierres et s'étendant dans le dernier tiers Sud du sondage (US 2207). À la fouille, celle-ci comprenait une quantité plus importante de tuiles et une quantité notable de céramique. Le long de la berme Ouest, un bloc taillé de grandes dimensions a été découvert. L'US 2207, de couleur marron, de consistance moyennement compacte (selon les endroits), était composée de nombreuses pierres de petit calibre et d'une quantité relativement plus importante de fragments de tuiles.

14 Au Sud du sondage, un mur de conception rudimentaire (sans mortier, pierres liées avec de la terre) a été découvert, sur un axe Ouest-Est, légèrement oblique. Selon le même axe, une bande de terre (US 2209) partant de la berme Ouest et identique à US 2210, se poursuivait sur tout le sondage (fig. 5). Au pied d'UC 2007 une forte concentration de mortier mélangé à de la terre a été découverte. Au niveau de la berme Ouest, la même concentration était présente au pied d'un ensemble de blocs qui semblaient constituer un mur Nord-Sud, comportant peut-être un retour Ouest-Est. Le mur rudimentaire au Sud du sondage présentait quelques similitudes avec les structures tardives apparentes sur le secteur $01:$ il pourrait donc s'agir d'une phase postérieure, peut-être en relation avec le « coup de sabre » visible dans UC 2007. On est donc potentiellement face à trois différentes phases de construction. Le matériel toutefois n'était pas significatif et était extrêmement mélangé. L'US 2205 présentait en outre une quantité anormalement importante de céramique archaïque. L'US 2207, quant à elle, contenait un tesson de céramique vraisemblablement médiévale.

Fig. 5 : Situation des US 2207-2211.

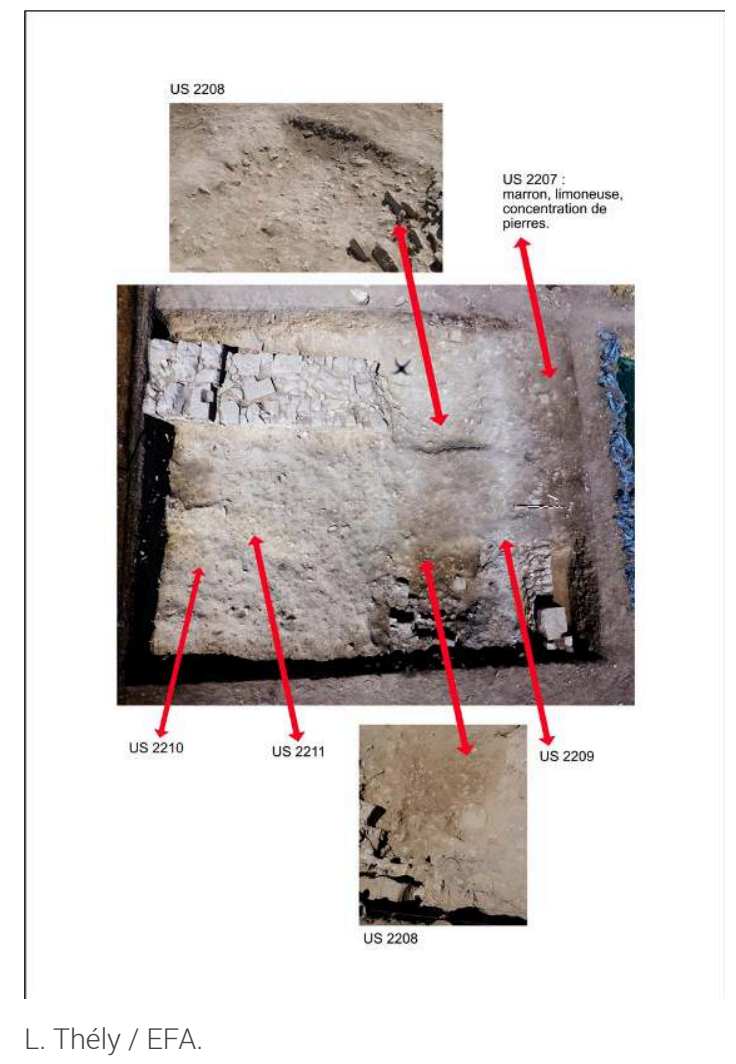

L'UC 2007 ne se poursuivait pas au Sud, mais il s'interrompait selon par un véritable arrachement (action moderne?). La morphologie des US du secteur pouvait correspondre à un ensemble d'interventions modernes. Les caractéristiques de 
l'US 2207 (le résultat d'un remblaiement moderne) semblaient confirmer cette hypothèse: un objet métallique oxydé, indéfini mais clairement moderne, y a été découvert entre autres. L'US 2207 recouvrait l'UC 2008: un mur d'apparence tardive, orienté Est-Ouest, légèrement en diagonale, formé d'un socle de petites pierres sur lesquelles reposait à l'Est un bloc taillé imposant.

Vers le centre du sondage, on a procédé ensuite à la fouille de l'US 2208, limoneuse, de couleur marron, qui contenait de nombreux fragments de mortier (fig. 6). Il s'est avéré dans un second temps que cette US n'était que l'interface entre US 2207 et plusieurs couches différentes. On a ainsi distingué trois situations (fig. 7) : à l'Ouest, à proximité des blocs en place émergeant, on distinguait une coupure assez nette et rectiligne en sens Nord-Sud qui semblait correspondre à la limite avec l'US 2211, à l'Est. Cette dernière était de couleur marron, argileuse et compacte. Enfin, sur une bande s'étendant approximativement sur la moitié Est du sondage, on a noté la présence d'une autre US, US 2210, grise et compacte, avec des petites inclusions de mortier, qui englobait des pierres dont une visiblement taillée et posée à plat. On a émis les deux hypothèses suivantes : soit ces trois couches forment des états différents (peut-être des moments différents) de la spoliation du mur Est-Ouest (UC 2008) ; soit US 2210 serait la trace du prolongement du mur Est-Ouest découvert en 2016 à l'Est, US 2211 constituerait une ouverture donnant un accès au Sud à cet espace, et US 2212 serait la trace des blocs manquants du mur Nord-Sud clôturant la pièce.

Fig. 6 : L'US 2208 ; au Nord, I'UC 2007, au Sud, I'UC 2008.

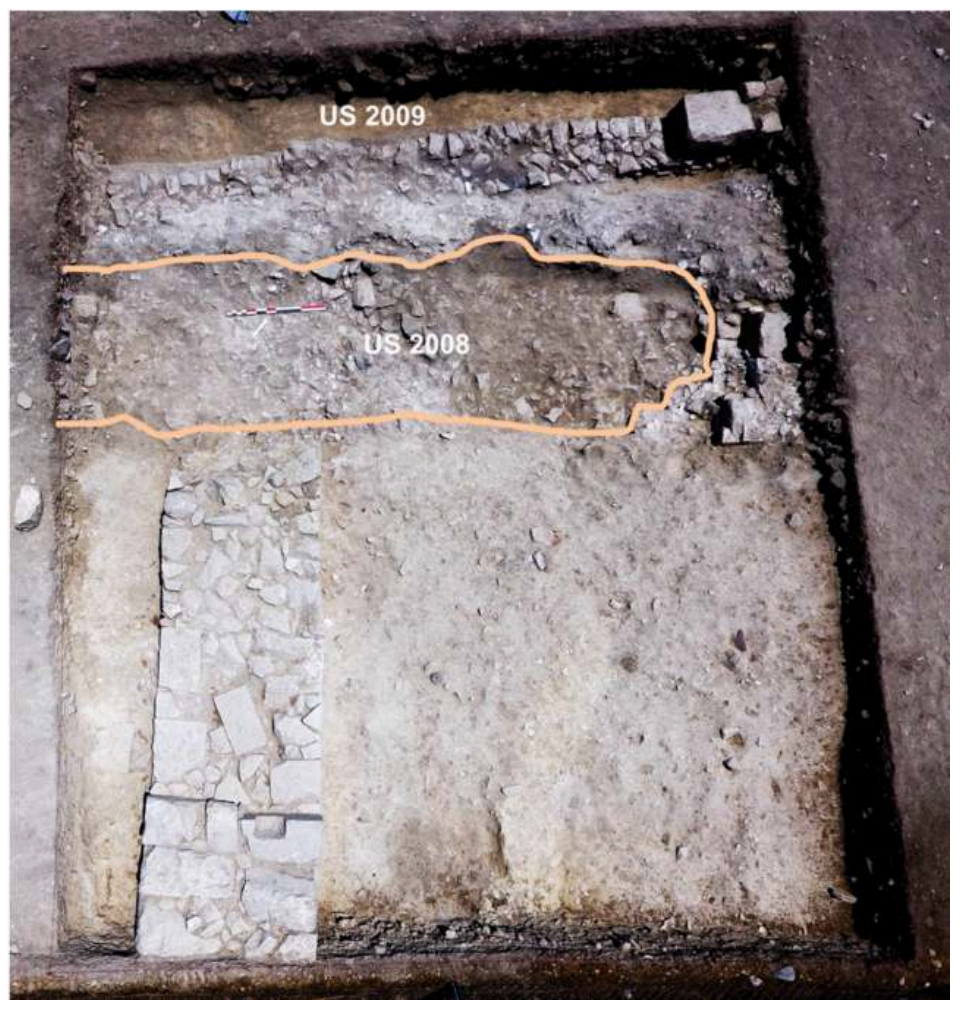

L. Thély / EFA. 
Fig. 7 : Situation des US 2210-2212.

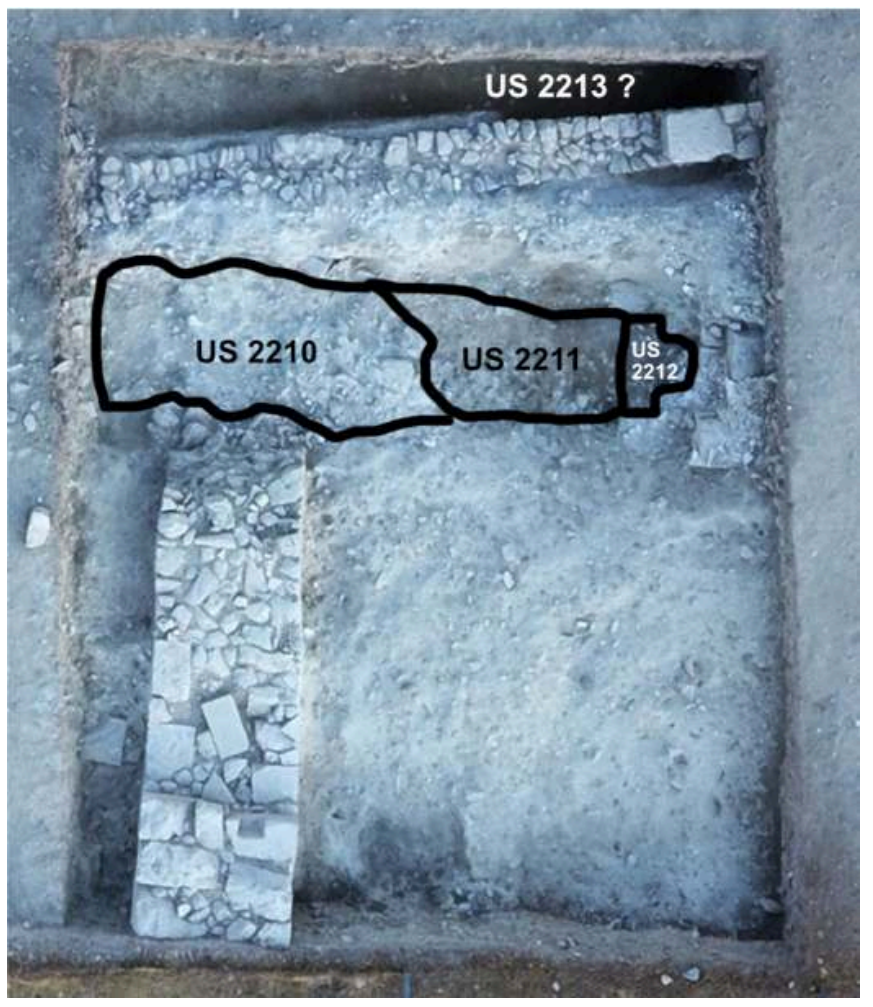

L. Thély / EFA.

17 Au Sud du sondage, l'US 2209 était une couche jaune sableuse, presque vierge de matériel, à tel point qu'on aurait pu l'interpréter comme du sable pur de chantier. Nous avons alors émis l'hypothèse qu'il pourrait s'agir du remplissage de la fosse de fondation du mur (tardif ?) UC 2008 dont les limites seraient rendues invisibles par la berme. Néanmoins, la fouille d'US 2209 a révélé qu'elle est en réalité posée sur une autre couche jaune, plus compacte, avec des inclusions de mortier. L'hypothèse de la tranchée de fondation est donc loin d'être certaine (sauf si l'on admet un double remplissage) d'autant que l'UC 2008 continue en profondeur.

Dans la structure qui affleure le long de la berme Ouest, on a observé un morceau de céramique pris dans du mortier. On retrouve la même technique en UC 2005 (campagne 2016) et de nombreux fragments répandus dans plusieurs couches. Cette technique de construction qui utilise un agglomérat de mortier et de céramique ne correspond pas forcément à la phase initiale de la structure, mais peut-être plutôt à une réparation tardive.

\section{Étude du matériel amphorique}

Antigone Marangou

Le matériel amphorique issu des campagnes de fouille menées en 2016 et 2017 aux abords Sud-Ouest de l'agora d'Amathonte a été étudié par A. Marangou, L. Pérès et N. Jaouen (Université de Rennes 2), à l'exception du matériel issu du secteur 1 en 2017. Les amphores ont été marquées, collées, comptées, identifiées et stockées dans la réserve selon leur centre de production. Les données chiffrées par US ont été 
enregistrées sous forme de tableaux qui résument la période chronologique avec ses principaux types amphoriques, le nombre des tessons et le Nombre Minimum d'Individus enregistrés. Les comptages ont été effectués systématiquement sur l'ensemble du matériel à l'exception des US de surface et des premières couches qui renfermaient un matériel très fragmentaire et usé, ce qui ne permettait pas de reconnaître la forme ou le type. Les dessins du matériel ont été réalisés par J. Humbert.

\section{BIBLIOGRAPHIE}

THÉLY 2015-2016

Ludovic Thély, « Inscriptions d'Amathonte XI. Un autel en l'honneur de Ptolémée X et

Bérénice III découvert aux abords Sud-Ouest de l'agora », BCH 139-140, 2015-2016, p. 463-484.

THÉLY et al. 2015-2016

Ludovic Thély, Julien Adam, Camille Castres, Antoine Chabrol, François-Dominique Deltenre, Antigone Marangou, Cécile Rocheron, « Rapport sur les travaux de l'École française d'Athènes en 2014 et en 2015 : Amathonte. Les abords Sud-Ouest de l'agora », BCH 139-140, 2015-2016, p. 980-1016.

THÉLY et al. à paraître

Ludovic Thély, Julien Adam, Dominique Barcat, Camille Castres, François-Dominique Deltenre, Antigone Marangou, Cécile Rocheron, « Amathonte : les abords Sud-Ouest de l'agora, 2016 », Bulletin archéologique des Écoles françaises à l'étranger.

\section{NOTES}

1. Chronique des fouilles en ligne notice $n^{\circ} 5067$, mise en ligne le 10 septembre 2015 , consultée le 29 juin 2020, https://chronique.efa.gr/?kroute=report\&id=5067 ; Chronique des fouilles en ligne notice $\mathrm{n}^{\circ} 5427$, mise en ligne le 5 juillet 2016, consultée le 29 juin 2020, https://chronique.efa.gr/?kroute=report\&id=5427; THÉLY et al. 2015-2016, p. 984-987.

2. THÉLY et al. 2015-2016 ; THÉLY et al. à paraître.

3. On fait allusion à la dédicace en l'honneur de Ptolémée X et Bérénice III : THÉLY 2015-2016. 


\section{INDEX}

\section{Thèmes : EFA}

chronologie https://ark.frantiq.fr/ark:/26678/pcrtpcAdBe2SFu

lieux https://ark.frantiq.fr/ark:/26678/pcrtrvSIfnFAUb

sujets https://ark.frantiq.fr/ark:/26678/pcrtwh5icnwutJ, https://ark.frantiq.fr/ark:/26678/ pcrt4HEc49rMp7, https://ark.frantiq.fr/ark:/26678/pcrtUtcfIvQKcB

Année de l'opération : 2017

\section{AUTEURS}

\section{LUDOVIC THÉLY}

Ministère de l'Europe et des Affaires étrangères

\section{JULIEN ADAM}

Université de Namur

\section{MARIE-HÉLÈNE BARRIÈRE}

UMR 5648 « Histoire, Archéologie, Littératures des mondes chrétiens et musulmans médiévaux (CIHAM)»

\section{CAMILLE CASTRES}

USR 3155 « Institut de recherche sur l'architecture antique (IRAA) »

FRANÇOIS-DOMINIQUE DELTENRE

École française d'Athènes

\section{ANTIGONE MARANGOU}

Université de Rennes 2

\section{RACHEL NOUET}

Université de Strasbourg

\section{CÉCILE ROCHERON}

UMR 8546 « Archéologie et Philologie d'Orient et d'Occident (AOROC) » 